\title{
Strangeness neutrality and baryon-strangeness correlations
}

\author{
Wei-jie Fu, ${ }^{1}$ Jan M. Pawlowski, ${ }^{2}$ and Fabian Rennecke $\oplus^{3, *}$ \\ ${ }^{1}$ School of Physics, Dalian University of Technology, Dalian 116024, People's Republic of China \\ ${ }^{2}$ Institut für Theoretische Physik, Universität Heidelberg, \\ Philosophenweg 16, 69120 Heidelberg, Germany \\ ${ }^{3}$ Physics Department, Brookhaven National Laboratory, Upton, New York 11973, USA
}

(Received 24 October 2018; published 20 December 2019)

\begin{abstract}
We derive a simple relation between strangeness neutrality and baryon-strangeness correlations. In heavy-ion collisions, the former is a consequence of quark number conservation of the strong interactions while the latter are sensitive probes of the character of QCD matter. This relation allows us to directly extract baryon-strangeness correlations from the strangeness chemical potential at strangeness neutrality. The explicit calculations are performed within a low-energy theory of QCD with $2+1$ dynamical quark flavors at finite temperature and density. Nonperturbative quark and hadron fluctuations are taken into account within the functional renormalization group. The results show the pronounced sensitivity of baryon-strangeness correlations on the QCD phase transition and the crucial role that strangeness neutrality plays for this observable.
\end{abstract}

DOI: 10.1103/PhysRevD.100.111501

\section{INTRODUCTION}

Extracting the phase diagram of QCD as a function of temperature $T$ and baryon chemical potential $\mu_{B}$ from hadronic final states is a main goal but also a main challenge in ultrarelativistic heavy-ion collisions. A detailed understanding of QCD matter in the hot and dense medium is indispensable for the interpretation and prediction of experimental data. The situation is further complicated by the fact that under the conditions of a heavy-ion collision, the phase diagram is not only spanned by $T$ and $\mu_{B}$, but for instance also other chemical potentials, electromagnetic fields, and various timescales. This is relevant for beam-energy scan experiments aiming at exploring the QCD phase diagram [1]. Fortunately, conservation laws can help to constrain some of these parameters.

The hadrons reaching the detectors in heavy-ion experiments inherit the properties of the QCD medium at freezeout. Since the typical freeze-out time is many orders of magnitude shorter than the timescale of flavor-changing weak interactions, the net quark number conservation of the strong interactions has to be taken into account. Consequently, there is a chemical potential associated to the conserved quark number of each quark flavor.

\footnotetext{
*frennecke@bnl.gov
}

Published by the American Physical Society under the terms of the Creative Commons Attribution 4.0 International license. Further distribution of this work must maintain attribution to the author(s) and the published article's title, journal citation, and DOI. Funded by SCOAP.
Focusing on the three lightest flavors, up $u$, down $d$, and strange $s$, the associated chemical potentials are conventionally given by linear combinations of baryon, charge, and strangeness chemical potentials $\mu_{B}, \mu_{Q}$, and $\mu_{S}$. With the chemical potential flavor-matrix

$\mu=\operatorname{diag}\left(\frac{1}{3} \mu_{B}+\frac{2}{3} \mu_{Q}, \frac{1}{3} \mu_{B}-\frac{1}{3} \mu_{Q}, \frac{1}{3} \mu_{B}-\frac{1}{3} \mu_{Q}-\mu_{S}\right)$,

their coupling to the quarks, $q=(u, d, s)^{T}$, is given by $\bar{q} \gamma_{0} \mu q$. In this work, we want to focus on strangeness and baryon number and therefore assume $\mu_{Q}=0$ for the sake of simplicity. This corresponds to isospin symmetric matter. Since the incident nuclei do not carry strangeness, the netstrangeness $\langle S\rangle \sim\left\langle\bar{s} \gamma_{0} s\right\rangle$ is fixed from the initial conditions of the collision. The condition $\langle S\rangle=0$ is called strangeness neutrality. Due to the peculiar beam-energy dependence of the net-baryon rapidity spectrum, the net-baryon number density $\langle B\rangle \sim\left\langle\bar{q} \gamma_{0} q\right\rangle$ in the quark-gluon plasma at central rapidities depends on the beam energy; see, e.g., [2]. We therefore work with the standard assumption that $\mu_{B}$ is a parameter we may choose freely, while $\mu_{S}$ is fixed through quark number conservation.

Strangeness is particularly interesting since strange particles are only created by collisions in the first place. This makes them valuable probes of the matter created in heavy-ion collisions [3]. In a recent work [4], we have investigated the effect of imposing strangeness neutrality on the phase structure and thermodynamics of QCD. Strangeness neutrality has a sizable impact on the QCD equation of state and the phase diagram, owing to an intricate interplay of strangeness coupled to meson-, baryon-, and quark dynamics at finite $T$ and $\mu_{B}$. 
Furthermore, this interplay also leads to the observation that a finite $\mu_{S}$ is required to enforce $\langle S\rangle=0$. Hence, we are led to the implicitly defined function,

$$
\mu_{S 0}\left(T, \mu_{B}\right)=\left.\mu_{S}\left(T, \mu_{B}\right)\right|_{\langle S\rangle=0} .
$$

Since $\mu_{B}$ couples to all quark flavors equally, increasing $\mu_{B}$ also increases the number of strange quarks over antistrange quarks. To ensure strangeness neutrality, a finite $\mu_{S}$ is necessary for compensation; see also, e.g., [5]. In the hadronic phase, the dominant degrees of freedom (d.o.f.) are either open strange mesons or strange baryons, depending on $\mu_{B}$. But while both couple to $\mu_{S}$, only the latter couple to $\mu_{B}$. Thus, it is intuitively clear that $\mu_{S 0}$ is a nontrivial function that is intimately tied to the nature of QCD matter.

In this paper, we will demonstrate that this deep connection between strangeness neutrality, which is a consequence of quark number conservation, and the dynamical interplay of hadrons and quarks, which is interweaved with the phase structure of QCD, can be made explicit. This is achieved by establishing an exact relation between $\mu_{S 0}$ and the baryon-strangeness correlation $C_{\mathrm{BS}}$; see (5). This correlation has been introduced in [6] as a sensitive probe of the nature of QCD matter. We will exploit said relation to compute $C_{\mathrm{BS}}$ at various $T$ and $\mu_{B}$ and carve out the important role that strangeness neutrality plays for this quantity. To this end, we employ a Polyakov loop enhanced quark-meson model (PQM) with $2+1$ dynamical quark flavors as a low-energy effective theory of QCD. Nonperturbative quantum, thermal and density fluctuations are taken into account with the functional renormalization group. Within this approach, quark-meson models are naturally embedded in QCD [7].

\section{STRANGENESS NEUTRALITY AND BARYON-STRANGENESS CORRELATIONS}

Generalized susceptibilities of conserved charges play a central role for theoretical and experimental studies of the QCD phase structure. This is due to the fact that the closely related cumulants of particle number distributions are directly sensitive to the growing correlation length at the phase transition [8]. In the present context at $\mu_{Q}=0$, the generalized susceptibilities are defined as chemical potential derivatives of the pressure $p$,

$$
\chi_{i j}^{\mathrm{BS}}\left(T, \mu_{B}, \mu_{S}\right)=\frac{\partial^{i+j} p\left(T, \mu_{B}, \mu_{S}\right) / T^{4}}{\partial \hat{\mu}_{B}^{i} \partial \hat{\mu}_{S}^{j}},
$$

with $\hat{\mu}=\mu / T$. Baryon number and strangeness are then given by

$$
\begin{aligned}
& \langle B\rangle=\left\langle N_{B}-N_{\bar{B}}\right\rangle=\chi_{1}^{B} V T^{3}, \\
& \langle S\rangle=\left\langle N_{\bar{S}}-N_{S}\right\rangle=\chi_{1}^{S} V T^{3},
\end{aligned}
$$

where $V$ is the spatial volume. We drop the superscript of the susceptibilities if the corresponding subscript is zero. The baryon-strangeness correlation $C_{\mathrm{BS}}$ [6] relevant for the present work reads at strangeness neutrality

$$
C_{\mathrm{BS}}\left(T, \mu_{B}, \mu_{S 0}\right)=-3 \frac{\langle\mathrm{BS}\rangle}{\left\langle S^{2}\right\rangle}=-3 \frac{\chi_{11}^{\mathrm{BS}}\left(T, \mu_{B}, \mu_{S 0}\right)}{\chi_{2}^{S}\left(T, \mu_{B}, \mu_{S 0}\right)} .
$$

Since the pressure is a function of $T, \mu_{B}$, and $\mu_{S}$, requiring strangeness neutrality implicitly fixes one of these variables as function of the others. Here, we choose the strangeness chemical potential, giving rise to Eq. (2). $\langle S\rangle=0$ implies

$$
\begin{aligned}
0 & =\frac{d}{d \hat{\mu}_{B}} \chi_{1}^{S}\left(T, \mu_{B}, \mu_{S 0}\right) \\
& =\chi_{11}^{\mathrm{BS}}\left(T, \mu_{B}, \mu_{S 0}\right)+\chi_{2}^{S}\left(T, \mu_{B}, \mu_{S 0}\right) \frac{\partial \hat{\mu}_{S 0}}{\partial \hat{\mu}_{B}} .
\end{aligned}
$$

This simple equation implicitly defines $\mu_{S 0}$ and we arrive at our central result,

$$
\frac{\partial \mu_{S 0}\left(T, \mu_{B}\right)}{\partial \mu_{B}}=\frac{1}{3} C_{\mathrm{BS}}\left(T, \mu_{B}, \mu_{S 0}\right) .
$$

Hence, together with the initial condition $\mu_{S 0}\left(\mu_{B}=0\right)=0$, $\mu_{S 0}$ can be extracted directly from $C_{\mathrm{BS}}$ at strangeness neutrality. By integrating the experimentally measured $C_{\mathrm{BS}}$ over the beam energy, one can extract the strangeness chemical potential at the freeze-out for isospin symmetric matter. Conversely, $C_{\mathrm{BS}}$ is given by the slope of $\mu_{S 0}\left(\mu_{B}\right)$. Most strikingly, $C_{\mathrm{BS}}$ has been introduced in [6] as a diagnostic for the nature of QCD matter. As argued in this work, this can be understood by explicitly examining $C_{\mathrm{BS}}$ at strangeness neutrality; see (5). Assuming that the system is deep in the deconfined regime, all strangeness is carried by $s$ and $\bar{s}$ and there is a strict relation between the baryon number carried by strange particles, $B_{s}$, and strangeness, $B_{s}=S / 3$. Furthermore, due to asymptotic freedom, there is no correlation between different quark flavors in this regime; it is a system of dilute current quarks. Equation (5) then implies $C_{\mathrm{BS}}=1$.

The situation is drastically different in the confined phase. Baryons can carry both baryon number and strangeness, while mesons can only carry strangeness. Thus, the denominator in Eq. (5), $\chi_{2}^{S}$, includes both open strange mesons and baryons, while the numerator, $\chi_{11}^{B S}$, only includes strange baryons. Hence, one generally finds $C_{\mathrm{BS}} \neq$ 1 in the confined hadronic phase. For $C_{\mathrm{BS}}<1$, the system is dominated by the fluctuations of open strange mesons and for $C_{\mathrm{BS}}>1$ it is dominated by strange baryons. Since cumulants of net particle numbers are experimental accessible, $C_{\mathrm{BS}}$ indeed serves as a sensitive probe of the composition of QCD matter, in particular of its strangeness content $[9,10]$. It is remarkable that $C_{\mathrm{BS}}$ can be directly related to $\mu_{S 0}$ via strangeness neutrality with Eq. (7) for any 
$T$ and $\mu_{B}$. This establishes a direct connection between quark number conservation and the phases of QCD.

We note that the situation becomes a little more complicated at $\mu_{Q} \neq 0$. In this case, Eq. (3) trivially generalizes to $\chi_{i j k}^{\mathrm{BSQ}}$. In addition to $\chi_{1}^{S}=0$, quark number conservation also implies, for instance, $\chi_{1}^{Q} / \chi_{1}^{B}=r$, where $r$ is a constant. This also implicitly defines the function $\mu_{Q 0}\left(T, \mu_{B}\right)$ and leads to a generalized form of Eq. (7),

$$
\frac{\partial \mu_{S 0}}{\partial \mu_{B}}=\frac{1}{3} C_{\mathrm{BS}}-\frac{\chi_{11}^{Q S}}{\chi_{2}^{S}} \frac{\partial \mu_{Q 0}}{\partial \mu_{B}},
$$

with

$$
\frac{\partial \mu_{Q 0}}{\partial \mu_{B}}=\frac{\chi_{11}^{\mathrm{BS}}\left(\chi_{11}^{\mathrm{SQ}}-r \chi_{11}^{\mathrm{BS}}\right)-\chi_{2}^{S}\left(\chi_{11}^{\mathrm{BQ}}-r \chi_{2}^{B}\right)}{\chi_{2}^{\mathrm{S}}\left(\chi_{2}^{Q}-r \chi_{11}^{\mathrm{BQ}}\right)-\chi_{11}^{\mathrm{SQ}}\left(\chi_{11}^{\mathrm{SQ}}-r \chi_{11}^{\mathrm{BS}}\right)} .
$$

The dependence on $T$ and $\mu_{B}$ of all quantities above is implied. The right-hand side of these equations is given by ratios of different baryon, strangeness, and charge correlations and therefore can be interpreted in an analogous manner to the discussion above, cf. [11]. Furthermore, since the involved susceptibilities can be measured, our main conclusion is not altered for the isospin-asymmetric case. It is worth noting that Eq. (8) generalizes the relations used on the lattice to implement the freeze-out conditions in an expansion about $\mu_{B} / T=0$ to any $T$ and $\mu_{B}$ [12].

\section{LOW-ENERGY EFFECTIVE THEORY AND FLUCTUATIONS}

In the following, we compute baryon-strangeness correlations with the help of Eq. (7). The impact of strangeness neutrality at finite baryon chemical potential is studied within a low-energy effective theory of QCD as initiated in [4]. In order to capture the main features of strangeness, quantum, thermal, and density fluctuations of open strange mesons, strange baryons and quarks have to be taken into account. Since kaons are pseudo-Goldstone bosons of spontaneous chiral symmetry breaking, they are the most relevant strange d.o.f. in the mesonic sector. Moreover, chiral symmetry dictates that if kaons are included as effective low-energy d.o.f., all other mesons in the lowest scalar and pseudoscalar meson nonets have to be included as well. By coupling quarks to a uniform temporal gluon background field $\bar{A}_{0}=\bar{A}_{0}^{(3)} t^{3}+\bar{A}_{0}^{(8)} t^{8}$, with $t^{c} \in S U(3)$, (statistical) confinement is taken into account. Below the deconfinement transition temperature $T_{d}$ predominantly three-(anti-)quark states contribute and baryons, instead of quarks, are the prevailing fermionic d.o.f. below $T_{d}$. In total, this gives rise to a $2+1$ flavor $\mathrm{PQM}$ with the Euclidean effective action $(\beta=1 / T)$

$$
\begin{aligned}
\Gamma_{k}= & \int_{0}^{\beta} d x_{0} \int d^{3} x\left\{\bar{q}\left(\gamma_{\nu} D_{\nu}+\gamma_{\nu} C_{\nu}\right) q+h \bar{q} \Sigma_{5} q\right. \\
& \left.+\operatorname{tr}\left(\bar{D}_{\nu} \Sigma \cdot \bar{D}_{\nu} \Sigma^{\dagger}\right)+\tilde{U}_{k}(\Sigma)+U_{\text {glue }}(L, \bar{L})\right\} .
\end{aligned}
$$

Quantum, thermal, and density fluctuations of modes with Euclidean momenta $k \leq|p| \lesssim 1 \mathrm{GeV}$ have been integrated out. The gauge covariant derivative is $D_{\nu}=\partial_{\nu}-i g \delta_{\nu 0} \bar{A}_{0}$. The scalar and pseudoscalar mesons are encoded in the flavor matrix $\Sigma=T^{a}\left(\sigma_{a}+i \pi_{a}\right)$, where the $T^{a}$ generate $U\left(N_{f}\right)$, and $\Sigma_{5}=T^{a}\left(\sigma_{a}+i \gamma_{5} \pi_{a}\right)$; see, e.g., [13]. The couplings of quarks and mesons to the chemical potential $\mu$ in Eq. (1) is achieved by formally introducing the vector source $C_{\nu}=\delta_{\nu 0} \mu$ and defining the covariant derivative acting on the meson fields $\bar{D}_{\nu} \Sigma=\partial_{\nu} \Sigma+\left[C_{\nu}, \Sigma\right]$, [14].

Spontaneous chiral symmetry breaking is captured by the meson effective potential $\tilde{U}_{k}(\Sigma)$, which consists of a fully $U\left(N_{f}\right)_{L} \times U\left(N_{f}\right)_{R}$ symmetric part plus pieces that explicitly break chiral symmetry through finite current quark masses and $U(1)_{A}$ through the axial anomaly. The deconfinement phase transition is captured statistically by including an effective potential for the gluon background field $U_{\text {glue }}(L, \bar{L})$, formulated in terms of the order parameter fields for deconfinement, the Polyakov loops $L=\operatorname{tr}_{c} \exp \left(i g \bar{A}_{0} / T\right), \bar{L}=\operatorname{tr}_{c}\left[\exp \left(i g \bar{A}_{0} / T\right)\right]^{\dagger}$. The strategy of Polyakov loop enhanced effective models is to use a potential that is fitted to the lattice equation of state of Yang-Mills theory and to include the effects of dynamical quarks through the coupling to the gluonic background in the quark covariant derivative. For a recent review, see [15]. We use the parametrization of the Polyakov loop potential put forward in [16], since it captures the lowest-order Polyakov loop susceptibilities which directly contribute to the particle number susceptibilities [17].

Owing to the intricate interplay of meson, baryon, and quark dynamics that contribute to strangeness neutrality and, as a consequence of Eq. (7), also to $C_{\mathrm{BS}}$, accounting for fluctuations of these d.o.f. is indispensable. In [4], we demonstrated that open strange meson fluctuations are crucial for strangeness neutrality, exacting a treatment beyond mean-field. Here, this is achieved by solving the renormalization group flow equation for the effective action $\Gamma_{k}$ using the functional renormalization group (FRG) [18],

$$
\partial_{t} \Gamma_{k}=\frac{1}{2} \operatorname{Tr}\left[\left(\Gamma_{k}^{(2)}[\Phi]+R_{k}\right)^{-1} \partial_{t} R_{k}\right]
$$

with $t=\ln (k / \Lambda) . \Gamma_{k}^{(2)}[\Phi]$ is the matrix of second functional derivatives of the effective action with respect to the fields $\Phi=(q, \bar{q}, \Sigma, L, \bar{L}) . R_{k}$ implements infrared-regularization at momenta $p^{2} \approx k^{2}$, and the trace involves the integration over loop-momenta, the color-, flavor-, and spinor-traces as well as the sum over different particle species. Solving Eq. (10) amounts to successively integrating out fluctuations starting from the initial action $\Gamma_{k=\Lambda}$, with 
$\Lambda=900 \mathrm{MeV}$ in our case, down to the full quantum effective action $\Gamma_{k=0}$. The FRG provides a nonperturbative regularization and renormalization scheme for the resummation of an infinite class of Feynman diagrams; see, e.g., [19] for QCD-related reviews.

It was shown in [4] that this approach leads to a very good agreement with the results of lattice QCD for the equation of state at vanishing $\mu_{B}$ and finite $\mu_{B} / T$ within the region accessible on the lattice. For further technical details on the model and on the RG flow equations, we refer to this work. Since the main qualitative features relevant for strangeness dynamics are captured by this approach, we will use it to compute the strangeness chemical potential at strangeness neutrality, $\mu_{S 0}\left(T, \mu_{B}\right)$ and then use Eq. (7) to extract the baryon-strangeness correlation $C_{\mathrm{BS}}$.

\section{NUMERICAL RESULTS}

From the solution of the RG flow equation (10), we obtain the full quantum effective action $\Gamma_{0}[\Phi]$. With the solution of the quantum equation of motion $\left.\left(\delta \Gamma_{0}[\Phi] / \delta \Phi\right)\right|_{\Phi=\Phi_{\mathrm{EOM}}}=0$, the pressure is given by $p=-\Gamma_{0}\left[\Phi_{\mathrm{EoM}}\right] / \beta V$. With this we are in the position to extract the generalized susceptibilities according to Eq. (3). First, we compute the strangeness number $\langle S\rangle$ in Eq. (3) as a function of $T, \mu_{B}$, and $\mu_{S}$. It turns out that for any $T$ and $\mu_{B}$ it is always possible to find a $\mu_{S}=\mu_{S 0}$ such that $\langle S\rangle\left(T, \mu_{B}, \mu_{S}=\mu_{S 0}\right)=0$. We restrict ourselves to $\mu_{B} \leq$ $675 \mathrm{MeV}$ since our model fails to capture important qualitative features of the theory at larger $\mu_{B}$, cf. [4]. The transition is a crossover in this range. The resulting $\mu_{S 0}$ is shown in Fig. 1. The characteristic shape of $\mu_{S 0}\left(\mu_{B}\right)$ can be understood qualitatively from our discussion of Eq. (7). At small $\mu_{B}$ strangeness is dominated by open strange mesons, so $C_{\mathrm{BS}}<1$ and hence $\mu_{S 0}\left(\mu_{B}\right)$ has a slope smaller



FIG. 1. Strangeness chemical potential at strangeness neutrality, $\mu_{S 0}$, as a function of the baryon chemical potential $\mu_{B}$ for various temperatures $T$ (solid lines). $\mathrm{T}$ is increasing from bottom to top from 100 to $250 \mathrm{MeV}$. The dashed line corresponds to the asymptotic limit of free quarks. In this limit, one finds $C_{\mathrm{BS}}=1$ which leads to $\mu_{S 0}=\mu_{B} / 3$ according to Eq. (7). than $1 / 3$. At larger $\mu_{B}$, strange baryons become dominant resulting in a slope larger than $1 / 3$. At large temperatures, the system undergoes a crossover to the deconfined phase with a pseudocritical temperature of $T_{d} \approx 155 \mathrm{MeV}$ in our model. Asymptotically, $\mu_{S 0}\left(\mu_{B}\right)$ approaches the dashed black line. However, since the Polyakov loops are still smaller than one at $T=250 \mathrm{MeV}$ in our computations, implying that the system is not fully deconfined, this asymptotic limit is not fully reached here. Still $\mu_{S 0}\left(\mu_{B}\right)$ is approximately linear already at $T \approx 180 \mathrm{MeV}$, with a slope only slightly smaller than $1 / 3$.

We extract the baryon-strangeness correlations $C_{\mathrm{BS}}$ from our result in Fig. 1 via Eq. (7). This is shown in Fig. 2. We find good agreement with the results of lattice QCD at vanishing $\mu_{B}$, highlighting that we capture the relevant effects quite accurately. Following our discussion above, we see that with increasing $\mu_{B}$ in the hadronic phase the baryon-strangeness correlations change from being dominated by the dynamics of open strange mesons to being dominated by strange baryons. At $\mu_{B} \gtrsim 420 \mathrm{MeV}, C_{\mathrm{BS}}(T)$ develops a nonmonotonicity. It first grows with $T$ since with increasing temperature more strange baryons can be excited and a larger $\mu_{S}$ has to be chosen in order to enforce strangeness neutrality. The resulting increasing slope of $\mu_{S 0}$ then directly translates to a rising $C_{\mathrm{BS}}$ through Eq. (7). For temperatures above the pseudocritical transition temperature, quark dynamics eventually take over, driving the system toward its asymptotic limit.

As a result of this dynamical interplay, $C_{\mathrm{BS}}(T)$ shows nonmonotonous behavior and develops a pronounced maximum already at moderate $\mu_{B}$. The sharper the crossover, the stronger this effect becomes. The maximum is



FIG. 2. Baryon-strangeness correlation $C_{\mathrm{BS}}$ as a function of temperature $T$ for different baryon chemical potential $\mu_{B}$ at strangeness neutrality. $\mu_{B}$ increases from 0 to $675 \mathrm{MeV}$ from bottom to top. We compare our results to the hadron resonance gas (HRG) containing only experimentally observed resonances [20]. At $\mu_{B}=0$ we also compare to the result of lattice QCD $[9,10,21]$. The thin black line indicates the free quark limit. The errors reflect the $95 \%$ confidence level of a cubic spline interpolation of our numerical data. 


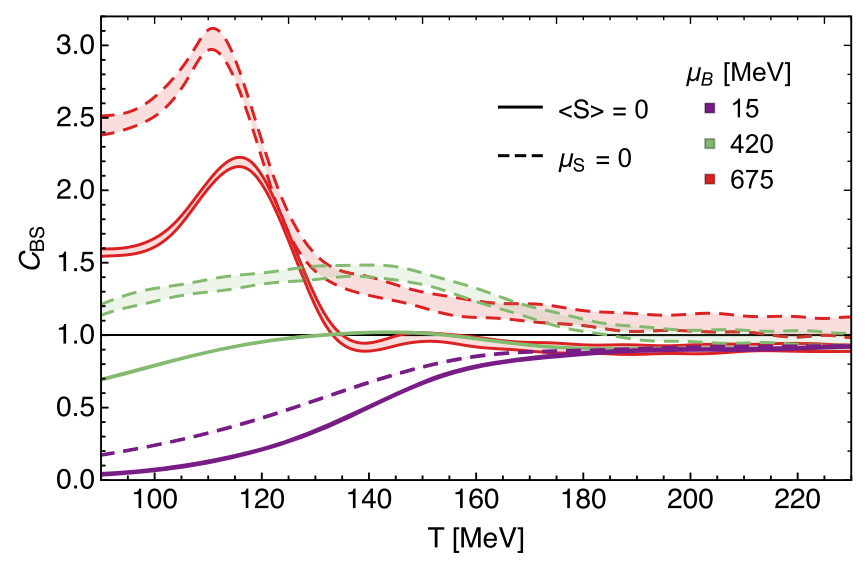

FIG. 3. Comparison between $C_{\mathrm{BS}}$ computed at strangeness neutrality, i.e., $\mu_{S}=\mu_{S 0}$ (solid lines), and at $\mu_{S}=0$ (dashed lines), where strangeness conservation is violated at finite $\mu_{B}$.

located exactly in the crossover region. We therefore find a distinct sensitivity of baryon-strangeness correlations to the chiral phase transition at finite $\mu_{B}$. This is potentially relevant for experimental measurements of $C_{\mathrm{BS}}$ : if the freeze-out is close to the phase transition, we predict a steep rise of $C_{\mathrm{BS}}$ with decreasing beam-energy.

As seen in Fig. 2, the HRG predicts monotonous behavior of $C_{\mathrm{BS}}(T)$ for any $\mu_{B}$ within the range of temperatures studied here. At large $\mu_{B}$, the nonmonotonous behavior we find leads to a significant enhancement over the HRG predictions at temperatures around the chiral crossover transition. This could be related to long-range strangeness dynamics close to the phase transition. In general, the HRG is in good agreement with our results well below the phase transition temperature. At $\mu_{B}=$ $300 \mathrm{MeV}$ and $420 \mathrm{MeV}$, we find good agreement also for larger temperatures. Whether this is physical or coincidental is not clear to us. In [10], it was argued that the discrepancy between the lattice and the HRG at $\mu_{B}=0$ could be due to yet undiscovered strange resonances which are not taken into account in the conventional HRG. So perhaps the effects of missing open strange mesons are compensated by baryon fluctuations at intermediate densities. In any case, this leads us to another prediction for the measurement of $C_{\mathrm{BS}}$ : it should be significantly enhanced over the HRG prediction at small beam-energies [22]. This would indicate a sharp crossover transition.
Finally, we want to explore the relevance of strangeness conservation on baryon-strangeness correlations. To this end, we compute $C_{\mathrm{BS}}$ also at vanishing strangeness chemical potential, $\mu_{S}=0$. Since there is no connection between $\mu_{S}$ and $C_{\mathrm{BS}}$ in this case, we have to compute $\chi_{11}^{\mathrm{BS}}$ and $\chi_{2}^{S}$ separately from the pressure. The result in comparison to the one at strangeness neutrality is shown in Fig. 3. We find a sizable enhancement of $C_{\mathrm{BS}}$ if strangeness neutrality is not taken into account, emphasizing the important role it plays here.

\section{SUMMARY}

We have shown that there is an intimate relation between particle number conservation and the QCD phase structure. This has been achieved by deriving a direct relation between baryon-strangeness correlations and strangeness neutrality. We explicitly demonstrated the sensitivity of $C_{\mathrm{BS}}$ on the phase structure and the relevance of strangeness neutrality for this observable. The study of the critical behavior of $C_{\mathrm{BS}}$ is deferred to future work. Since it is given by a ratio of second-order cumulants, it may not be sensitive to criticality at all. We emphasize that the sensitivity of $C_{\mathrm{BS}}$ to the phase transition observed here is due to the change from hadronic to partonic dynamics, which is more pronounced at larger $\mu_{B}$ due to the specific dynamics that drive $C_{\mathrm{BS}}$. A meaningful comparison between our theoretical prediction and experimental measurements may require, among other things, the description of net-kaons and protons instead of conserved charges, as well as nonequilibrium effects.

\section{ACKNOWLEDGMENTS}

We thank Robert D. Pisarski for discussions and Swagato Mukherjee for providing us with the lattice data for Fig. 2. F. R. is supported by the Deutsche Forschungsgemeinschaft (DFG) through Grant No. RE 4174/1-1. W. F. is supported by the National Natural Science Foundation of China under Contract No. 11775041. This work is supported by the ExtreMe Matter Institute. It is part of and supported by the DFG Collaborative Research Centre "SFB 1225 (ISOQUANT)".
[1] M. Gazdzicki, Z. Fodor, and G. Vesztergombi (NA49 Collaboration), Study of hadron production in hadron-nucleus and nucleus-nucleus collisions at the CERN SPS, CERN Technical Reports No. SPSC-P-330 and
No. CERN-SPSC-2006-034, 2006, revised version submitted on 2006-11-06; STAR Collaboration, Star note 598: Studying the phase diagram of QCD matter at RHIC (2014); B. Friman, C. Hohne, J. Knoll, S. Leupold, J. Randrup, 
R. Rapp, and P. Senger, Lect. Notes Phys. 814, 11 (2011); V. D. Kekelidze, R. Lednicky, V. A. Matveev, I. N. Meshkov, A. S. Sorin, and G. V. Trubnikov, Eur. Phys. J. A 52, 211 (2016); T. Galatyuk, Nucl. Phys. A931, 41 (2014); H. Sako et al., Nucl. Phys. A931, 1158 (2014).

[2] L. Adamczyk et al. (STAR Collaboration), Phys. Rev. C 96, 044904 (2017).

[3] P. Koch, B. Muller, and J. Rafelski, Phys. Rep. 142, 167 (1986).

[4] W.-j. Fu, J. M. Pawlowski, and F. Rennecke, arXiv: 1808.00410.

[5] J. Letessier, A. Tounsi, U. W. Heinz, J. Sollfrank, and J. Rafelski, Phys. Rev. D 51, 3408 (1995).

[6] V. Koch, A. Majumder, and J. Randrup, Phys. Rev. Lett. 95, 182301 (2005).

[7] M. Mitter, J. M. Pawlowski, and N. Strodthoff, Phys. Rev. D 91, 054035 (2015); J. Braun, L. Fister, J. M. Pawlowski, and F. Rennecke, Phys. Rev. D 94, 034016 (2016); F. Rennecke, Phys. Rev. D 92, 076012 (2015); A. K. Cyrol, M. Mitter, J. M. Pawlowski, and N. Strodthoff, Phys. Rev. D 97, 054006 (2018).

[8] M. A. Stephanov, Phys. Rev. Lett. 102, 032301 (2009).

[9] A. Bazavov et al., Phys. Rev. Lett. 111, 082301 (2013).

[10] A. Bazavov et al., Phys. Rev. Lett. 113, 072001 (2014).

[11] A. Majumder and B. Muller, Phys. Rev. C 74, 054901 (2006).

[12] A. Bazavov et al., Phys. Rev. Lett. 109, 192302 (2012); S. Borsanyi, Z. Fodor, S. D. Katz, S. Krieg, C. Ratti, and K. K. Szabo, Phys. Rev. Lett. 111, 062005 (2013); A. Bazavov et al. (HotQCD Collaboration), Phys. Rev. D 96, 074510 (2017).
[13] F. Rennecke and B.-J. Schaefer, Phys. Rev. D 96, 016009 (2017).

[14] J. B. Kogut and D. Toublan, Phys. Rev. D 64, 034007 (2001).

[15] K. Fukushima and V. Skokov, Prog. Part. Nucl. Phys. 96, 154 (2017).

[16] P. M. Lo, B. Friman, O. Kaczmarek, K. Redlich, and C. Sasaki, Phys. Rev. D 88, 074502 (2013).

[17] W.-j. Fu, J. M. Pawlowski, F. Rennecke, and B.-J. Schaefer, Phys. Rev. D 94, 116020 (2016).

[18] C. Wetterich, Phys. Lett. B 301, 90 (1993).

[19] J. Berges, N. Tetradis, and C. Wetterich, Phys. Rep. 363, 223 (2002); J. M. Pawlowski, Ann. Phys. (Amsterdam) 322, 2831 (2007); H. Gies, Lect. Notes Phys. 852, 287 (2012); B.-J. Schaefer and J. Wambach, Phys. Part. Nucl. 39, 1025 (2008); J. Braun, J. Phys. G 39, 033001 (2012).

[20] P. Braun-Munzinger, K. Redlich, and J. Stachel, in Quark-Gluon Plasma, edited by R. C. Hwa (World Scientific Publishing, Singapore, 2003), Vol. 3, pp. 491-599; M. Tanabashi et al. (Particle Data Group), Phys. Rev. D 98, 030001 (2018).

[21] A. Bazavov et al., Phys. Rev. D 95, 054504 (2017); A. Bazavov et al. (HotQCD Collaboration), Phys. Lett. B 795, 15 (2019); S. Mukherjee (private communication).

[22] We start seeing a pronounced peak forming at $\mu_{B} \gtrsim 550 \mathrm{MeV}$. If we take the scales of our computation at face value, this corresponds to beam energies of $\sqrt{s} \lesssim 5 \mathrm{GeV}$. This would be beyond the reach of current beam-energy scan experiments, but within the range covered by future experiments, e.g., at FAIR, NICA, or J-PARC. 